\title{
Investigating High Turbulent Flows by Laser Doppler Anemometry
}

\author{
${ }^{1}$ Ilnar F. Ramazanov, ${ }^{2}$ Farit F. Ramazanov, ${ }^{3}$ Leysan F. Ryadninskaya \\ ${ }^{1-3}$ Kazan Federal University \\ Email:kafiziki@mail.ru
}

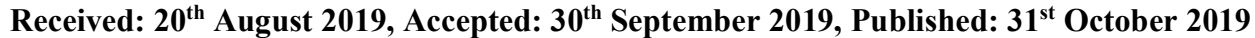

\begin{abstract}
The article discusses the use of a laser Doppler anemometer for measuring flow parameters with a high degree of turbulence in power plants.

Due to the lack of rigorous analytical solutions of the equations describing the turbulent flow regime of liquid and gas flows, the use of experimental methods for studying flows is an urgent scientific task. The precision method is the optical method of laser Doppler anemometry, with which you can determine the speed of flow.

An automated system based on a two-color argon laser has been developed, which allows one to determine the flow velocity in two orthogonal planes with the determination of the sign of the velocity projection in the speed range from 0 to $\pm 150 \mathrm{~m} / \mathrm{s}$. The error of speed measurement is from 0.1 to $3 \%$. Carrying out two series of measurements of the velocity projection at the same point in the flow allows us to determine the magnitude and direction of the velocity vector. The measuring volume is an ellipsoid of revolution with a section diameter of $144 \mu \mathrm{m}$. The flow velocity was measured in two sections of the cylinder of the internal combustion engine, as well as in the valve slit.

During the study, it was revealed that the non-contact method of laser Doppler anemometry allows you to get a reliable picture of the distribution of the velocity field in the cylinder of an internal combustion engine.

\section{Keywords}

Laser Doppler Anemometer, Light Scattering Particles, Measuring Volume, Detection-Estimation Algorithm, Velocity Field.

\section{Introduction}

The measurement of fluid or gas flow rates with a high degree of turbulence is an urgent scientific problem due to the lack of rigorous analytical solutions. Therefore, experimental methods for studying turbulent flows are of particular importance. An absolute method for measuring the flow velocity is the laser Doppler anemometry (LDA) method described in the literature [1-3]. By measuring the Doppler frequency shift (DFS) caused by the scattering of light by optical inhomogeneities - light scattering particles (MF), one can determine the flow velocity, since there is an unambiguous, linear relationship between the DFS and the particle velocity. At subsonic flow velocities, the relative change in the radiation frequency is of the order of 10-8; therefore, the use of the Doppler effect for measuring flow velocities is possible only by scattering monochromatic laser radiation from optical inhomogeneities.

In the study of turbulent flows of liquid and gas, it is not enough to know the average flow velocity and velocity pulsations; it is also necessary to determine the direction of the flow velocity vector. Therefore, to measure the speed of the midrange, two or three-component LDAs are used. Three-component LDAs are technically difficult to implement. Two-component LDAs are the most optimal, although they also have a significant drawback - to determine the orientation of the velocity vector at each point of the flow, it is necessary to measure the velocity in two planes.
\end{abstract}

\section{Methods}

To determine the two components of the flow velocity, two lines of argon laser radiation are used. In this case, two orthogonal systems of interference bands formed by two light waves of different frequencies are formed in the measuring volume (IO). To determine the sign of two velocity projections, a Bragg cell is used. An LDAbased system [8,9] is used for single-point measurements in liquid and gas flows with an unknown flow direction with a high degree of turbulence.

In a two-component LDA, a measuring volume is formed when two pairs of orthogonally spaced beams are superimposed. If at some point in space two light waves with slightly different frequencies are combined $v 1$ and $v 2(\Delta v=v 1-v 2<<1+v 2)$ : $E_{1}=E_{0} \cos \left(2 \pi v_{1} t-k_{1} z\right)$ and $E_{2}=E_{0} \cos \left(2 \pi v_{2} t-k_{2} z\right)$,

where $\mathrm{k}_{1}$ and $\mathrm{k}_{1}$ are wave numbers, $\mathrm{z}$ is the coordinate of the point measured in the direction of the light waves, the resulting wave is formed:

$E=E_{1}+E_{1}=2 E_{0} \cos \left[2 \pi\left(v_{1}-v_{2}\right) t / 2-\left(k_{1}-k_{2}\right) z / 2\right] \cdot \cos \left[2 \pi\left(v_{1}+v_{2}\right) t / 2-\left(k_{1}+k_{2}\right) z / 2\right]$.

as $\left|v_{1}-v_{2}\right|<<v_{1}+v_{2}$, then the factor

$2 E_{0} \cos \left[2 \pi\left(v_{1}-v_{2}\right) t / 2-\left(k_{1}-k_{2}\right) z / 2\right]$

represents the slowly changing amplitude of the resulting wave. The rate of change of amplitude can be found by determining the phase velocity from the condition: 
$2 \pi\left(v_{1}-v_{2}\right) t / 2-\left(k_{1}-k_{2}\right) z / 2=$ const.

there: $\frac{d z}{d t}=\frac{2 \pi\left(v_{1}-v_{2}\right)}{k_{1}-k_{2}}$.

If the frequencies of the incident light waves are the same $\left(v_{1}=v_{2}\right)$, then $\frac{d z}{d t}=0$ and the working volume is a fixed interference pattern. If the frequencies of the two waves are different $\left(\Delta v_{1}=v_{1}-v_{2} \neq 0\right)$, then $\frac{d z}{d t}=V_{u n}=$ const and in the interference pattern observed in the IO, there is a continuous change of maxima to minima and vice versa, i.e. a running interference pattern is observed with the velocity of the $V_{\text {ip }}$ bands moving. In this case, it is possible to determine the direction of motion of the particle in the flow through the IO and, accordingly, the velocity vector.
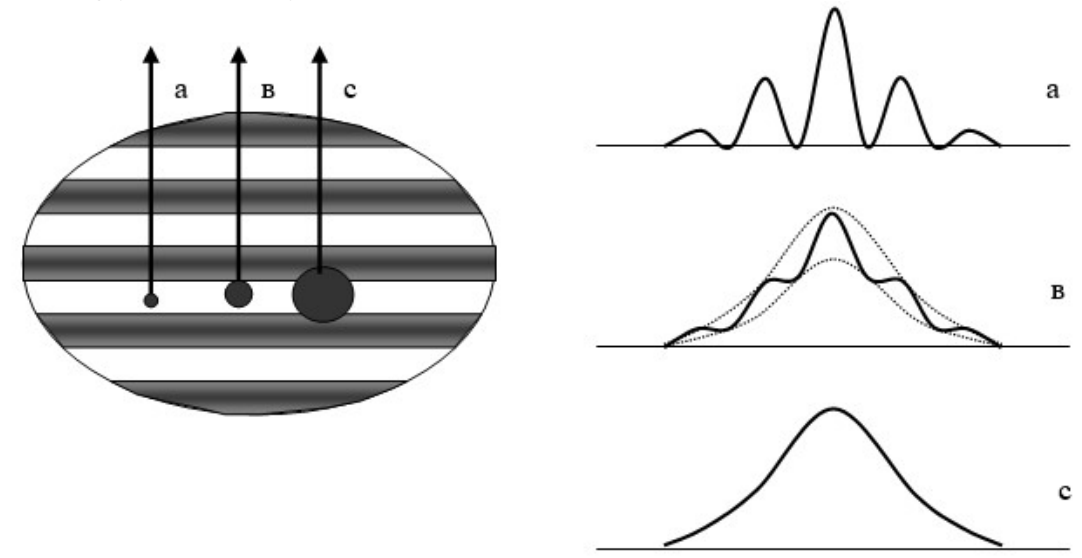

Fig 1: The Dependence of the Modulation Depth of the Signal on the Particle Size

A particle flying through a measuring volume, which is an ellipsoid of revolution (Fig. 1.), A particle flying through a measuring volume, which is an ellipsoid of revolution (Fig. 1.), intersects interference fringes and the intensity of the light scattered by it changes sinusoidally in time. The frequency of this change is a function of the frequency difference of two light rays $\Delta v$, the particle velocity $\vec{V}$ and the relative angle between the rays scattered by the particle, characterized by a difference wave vector $\vec{K}$ :

$$
\Delta v_{d}=v_{1}-v_{2}-\frac{\vec{V} \cdot \vec{K}}{2 \pi}
$$

where $\frac{\vec{V} \cdot \vec{K}}{2 \pi}=\Delta \nu_{d}-$ "true" Doppler frequency shift due to particle velocity $\vec{V}$.

$$
\text { If } v_{l}=v_{2} \text {, то } \Delta \boldsymbol{v}_{\boldsymbol{d}}^{\prime}=\Delta \boldsymbol{v}_{\boldsymbol{d}}=-\frac{\overrightarrow{\boldsymbol{V}} \cdot \overrightarrow{\boldsymbol{k}}}{2 \pi} \text {. }
$$

The processing system determines the absolute magnitude of the frequency change and as a result, it is possible to determine only the absolute value of the speed from the relation:

$$
\Delta v_{d}=\left|-\frac{\vec{V} \cdot \vec{K}}{2 \pi}\right|
$$

Thus, in the case $v 1=v 2$, irrespective of the direction of motion of the particle, the particle velocities that are identical in absolute value lead to the same value $\Delta v_{d}$.

If the frequencies of the two waves differ $v_{l} \neq v_{2}$, then

$$
\Delta v_{d}^{\prime}=\left(\Delta v-\frac{V^{\prime} \cdot K}{2 \pi}\right)
$$

In this case, the sign of speed (particle motion direction) will be determined by whether the value found by the photodetector will be $\Delta v_{d}^{T}$ more or less than $\Delta \boldsymbol{\nu}$.

Depending on whether the particle moves in the direction of movement of the interference fringes or in the opposite direction, the photodetector captures a different frequency. 
In particular, if $\vec{V}_{i p} \uparrow \uparrow \vec{V}, \Delta \boldsymbol{v}_{\boldsymbol{d}}^{\prime}<\Delta \boldsymbol{v}_{d}$. If $\vec{V}_{i p}^{+} \uparrow \downarrow \vec{V}$, then $\Delta v_{n}^{\prime}>\Delta v_{d}$. If the particle and interference fringes move in the same direction, the frequency is fixed. $\boldsymbol{\Delta} \boldsymbol{v}_{\boldsymbol{d}}^{\prime}<\left|\boldsymbol{\Delta} \boldsymbol{v}_{\boldsymbol{d}}\right|$. Otherwise $\Delta \boldsymbol{v}_{d}^{\prime}>\left|\Delta \boldsymbol{v}_{d}\right|$

The definition of only one velocity component, for example, $\mathrm{V}_{\mathrm{x}}$, is described above. The use of another pair of rays allows independent determination of another component of the velocity $\mathrm{V}_{\mathrm{y}}$.

The full value of the particle velocity in the XY plane is

$$
V=\sqrt{V_{x}^{2}+V_{y}^{2}}
$$

Thus, the determination of the direction of the velocity $\mathrm{V}$ by means of an LDA is possible if the frequency of light in one of the rays is different from the frequency in the other.

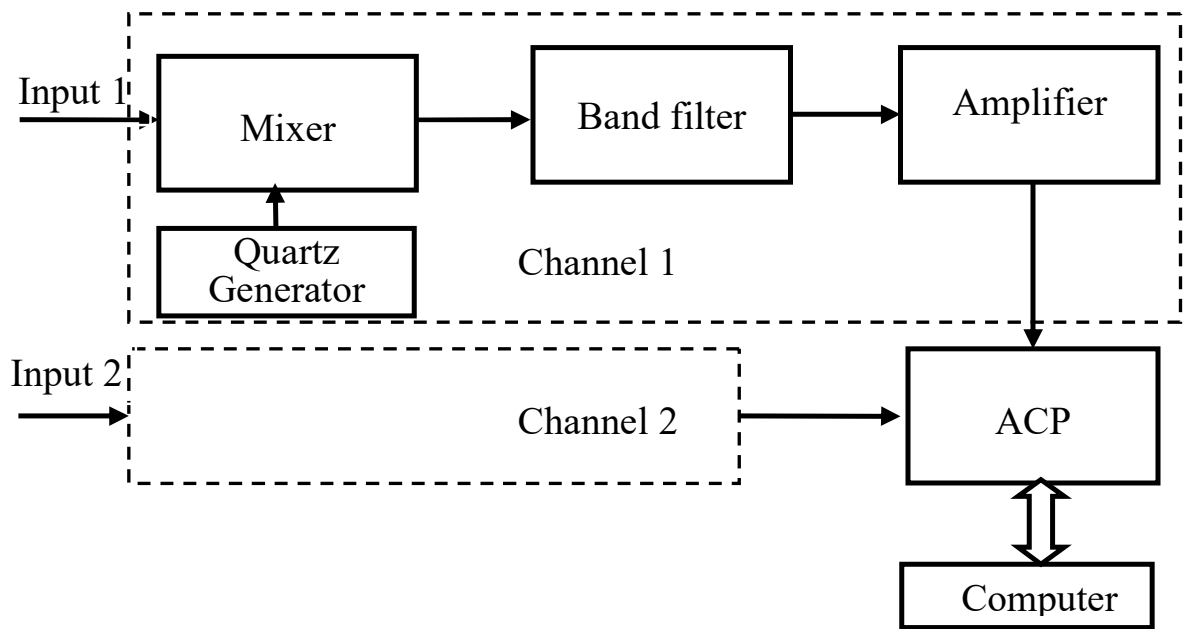

Fig 2: Functional Diagram of the Processing System

A description of the optical part of the research facility is given in [5-7].

To solve the problem of detecting and estimating the stochastic signal of an LDA, an analog-to-digital converter (ADC) can be used. In the processing system (Fig. 2.), the LDA signal is input in digital form into a computer and further processed [2].

To process the LDA signal, a system was developed based on the LA-n10M6 ADC with a sampling frequency of $100 \mathrm{MHz}$ for digital processing of the LDA signal in real time [3]. Functional diagram of the signal processing system is shown in Fig. 2.

The signal processing system according to [4] must distinguish between two competing hypotheses:

$\mathrm{H}_{0}: \xi(\mathrm{t})=\zeta\left(\mathrm{t}, v_{\mathrm{d}}\right)+\mathrm{n}(\mathrm{t})$,

$\mathrm{H}_{1}: \xi(\mathrm{t})=\mathrm{n}(\mathrm{t})$,

where $\xi(\mathrm{t})$ - processing input, $\zeta\left(\mathrm{t}, v_{\mathrm{d}}\right)$ LDA signal, $\mathrm{n}(\mathrm{t})$ - noize. According to $\mathrm{H} 0$, at the system input, a mixture of the useful signal and noise; according to H1, there is no useful signal at the system input. The presence of a signal already confirms the presence of useful information in it, because it is contained not only in the Doppler frequency $v_{\mathrm{d}}$, which is an informative parameter of the signal. The criterion for signal detection is the excess of the signal amplitude of a predetermined threshold value.

In the implemented system [7], the LDA signal frequency of $80 \pm 5 \mathrm{MHz}$ is reset to the region of $10 \pm 5 \mathrm{MHz}$ using a mixer. A 14th order elliptical filter filters the signal.

To study the velocity field of the aerodynamic flow in the cylinder of an internal combustion engine (ICE), a special block of a model cylinder was developed. The block consists of a cylinder model made of transparent material, as well as an AVL-2 cylinder head mounted on a rotary assembly. Thanks to the swivel unit, it is possible to change the orientation of the cylinder head to measure both longitudinal and transverse flow rates. To purge the cylinder, a special gas supply system was used: a compressor, a flow valve, a pressure gauge for measuring pressure, a midrange generator and pipelines. Using a compressor, air is supplied to the inlet of the head, while the gas flow can be simulated by changing the air flow in the head.

The concentration and particle size of natural dust are very small, so the amplitude of the reflected signal is insufficient for the sensitivity of the recording equipment used. Therefore, it is necessary to introduce light scattering particles into the studied stream to create artificial dust content of the stream.

Particles must meet the following requirements:

1) Must follow the airflow with the smallest speed defect. For this, the particles must have small size and density. The requirements of an aerodynamic experiment are met by particles with a diameter of the order of $d=$ $0.5 \ldots 2.0 \mu \mathrm{m}$. 
2) The diameter of the light-scattering particles must be consistent with the step $\Delta$ of interference fringes in the measuring volume: $\frac{d}{A}<<1$. If the particle diameter becomes comparable with the spacing of the bands, the signal modulation depth is sharply worsened.

1) Particles should not be excessively small in size, since the intensity of the scattered light decreases in proportion to the sixth power of the particle diameter, which sharply increases the sensitivity requirements for recording equipment.

2) The concentration should be sufficient to ensure the necessary information content of the measurements, but not too large, since an additional error arises due to the superposition of signals from two (or more) particles.

The optimal mode is a single-particle mode, when one particle is in the measuring volume. This mode in the developed installation is provided by a light-scattering particle generator, which serves to obtain a monodisperse aerosol with a given particle diameter and optimal concentration.

The diameter of light-scattering particles is determined from the condition of following along with the flux and from the condition of maximum modulation of the light flux. From the analysis of a number of works [3,4] it follows that the most efficient and at the same time convenient and simple is an aerosol generator with small ( 0.5 - $1.5 \mu \mathrm{m}$ ) solid particles that reflect light well. The main disadvantage of aerosols with solid particles is the coagulation of particles, which leads to polydispersity and inconsistent aerosol concentrations.

In the research setup, a mode is implemented in which there is one midrange in the IO. Thanks to the MF generator, this mode is ensured in the developed installation and monodisperse aerosol is obtained with a given particle diameter and optimal concentration. For research, a specially made $\mathrm{Al}_{2} \mathrm{O}_{3}$ powder was used. Monodisperse MFs had a diameter of $0.5 \mu \mathrm{m}$ and high reflectivity.

\section{Results and Discussion}

Thanks to the developed stand, studies of gas-dynamic flows in the engine cylinder at the engine test station of the Scientific and Technical Center of PJSC KAMAZ were carried out [10-12]. The measurements were carried out inside the model cylinder at a certain point in the flow in the static purge mode. The control of the experiment and the statistical processing was carried out by special software. The measurements were carried out twice at each point of the flow for different IO orientations in several sections of the ICE cylinder $(Z=20 \mathrm{~mm}$ and $Z=80 \mathrm{~mm}$ ), as well as in the valve slit. The results are presented in table form for the three components of the average velocity vector in polar coordinates.

The distribution of the vertical velocity component $v_{z}$ in the cylinder of the AVL -2 head at $Z=80 \mathrm{~mm}, \Delta p=$ $100 \mathrm{kPa}$ is shown in Fig. 3.

\begin{tabular}{|c|c|c|c|c|}
\hline № points & $\mathrm{v}_{\mathrm{r}}, \mathrm{m} / \mathrm{s}$ & $v_{\varphi .}, \mathrm{m} / \mathrm{s}$ & $\mathrm{v}_{\mathrm{z}}, \mathrm{m} / \mathrm{s}$ & $\mathrm{v}, \mathrm{m} / \mathrm{s}$ \\
\hline 1 & 07.43 & -17.94 & 16.86 & 25.72 \\
\hline 2 & 15.61 & -11.75 & 19.75 & 27.78 \\
\hline 3 & 20.92 & 02.91 & 11.10 & 23.86 \\
\hline 4 & 10.05 & -16.70 & 21.25 & 28.83 \\
\hline 5 & 09.05 & -28.91 & 26.11 & 39.99 \\
\hline 6 & 09.61 & -16.82 & 21.41 & 28.87 \\
\hline 7 & -09.90 & -07.63 & 18.41 & 22.22 \\
\hline 8 & -13.29 & -17.15 & 19.22 & 28.99 \\
\hline 9 & -03.23 & -14.84 & 22.19 & 26.89 \\
\hline 10 & -05.56 & -17.64 & 19.38 & 26.79 \\
\hline 11 & -03.38 & -39.90 & 27.55 & 48.60 \\
\hline 12 & -00.41 & -16.54 & 29.78 & 34.08 \\
\hline 13 & 05.75 & 02.35 & 22.29 & 23.14 \\
\hline 14 & -02.99 & -03.11 & 27.96 & 28.29 \\
\hline 15 & 11.64 & -19.36 & 25.28 & 33.90 \\
\hline 16 & -11.42 & -05.70 & 10.48 & 16.51 \\
\hline 17 & 04.27 & 03.70 & 32.65 & 33.14 \\
\hline 18 & -06.60 & -11.79 & 19.02 & 23.33 \\
\hline 19 & 01.81 & 05.44 & 05.74 & 08.11 \\
\hline 20 & -00.23 & -01.38 & 22.50 & 22.54 \\
\hline 21 & 01.88 & 04.72 & 07.53 & 09.08 \\
\hline 22 & -02.49 & 10.81 & 13.25 & 17.28 \\
\hline 23 & 00.08 & 00.53 & 07.28 & 07.30 \\
\hline 24 & 13.37 & -07.74 & 11.26 & 19.12 \\
\hline 25 & 13.84 & -08.81 & 12.11 & 19.12 \\
\hline
\end{tabular}

Table 1: Components of Speed in the Cylinder Head AVL - 2 at $Z=80 \mathrm{~mm}, \Delta p=100 \mathrm{kPa}$. 


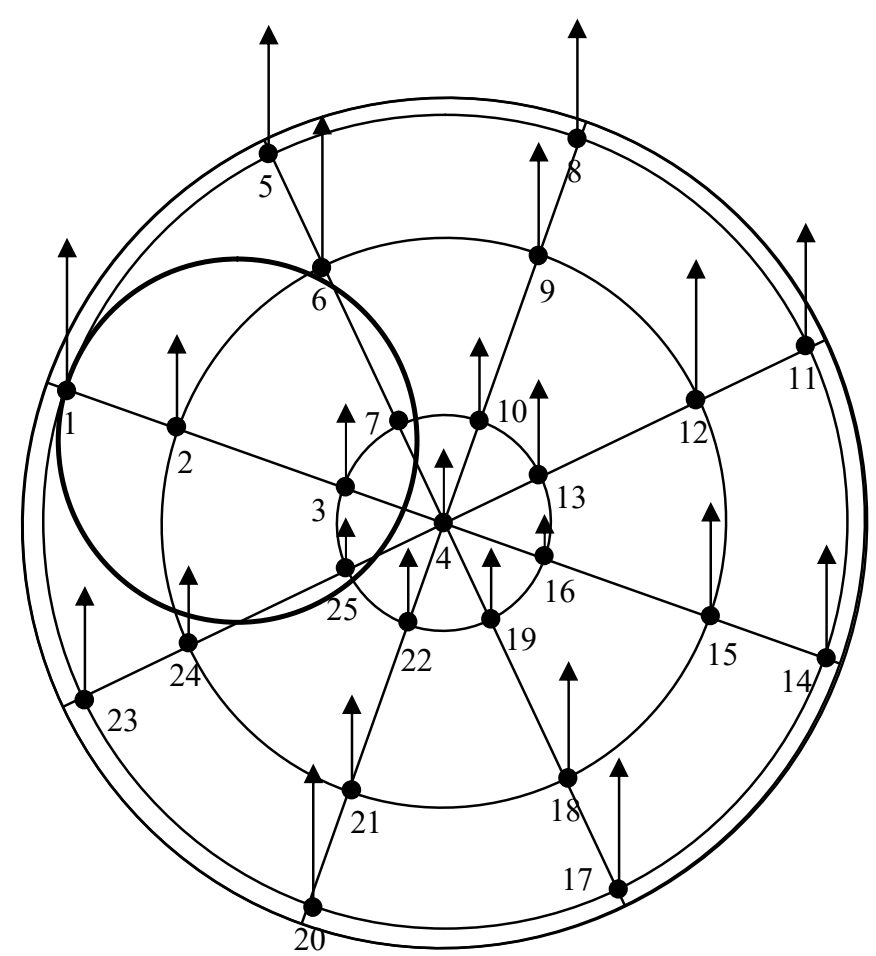

Fig. 3: The Distribution of the Vertical Component of the Velocity in the Cylinder Head AVL - 2 at $Z=80$ $\mathrm{mm}, \Delta \mathrm{p}=100 \mathrm{kPa}$.

The distribution of the horizontal velocity component in the cylinder of the AVL - 2 head at $Z=80 \mathrm{~mm}, \Delta \mathrm{p}=$ $100 \mathrm{kPa}$ is shown in Fig. 4.

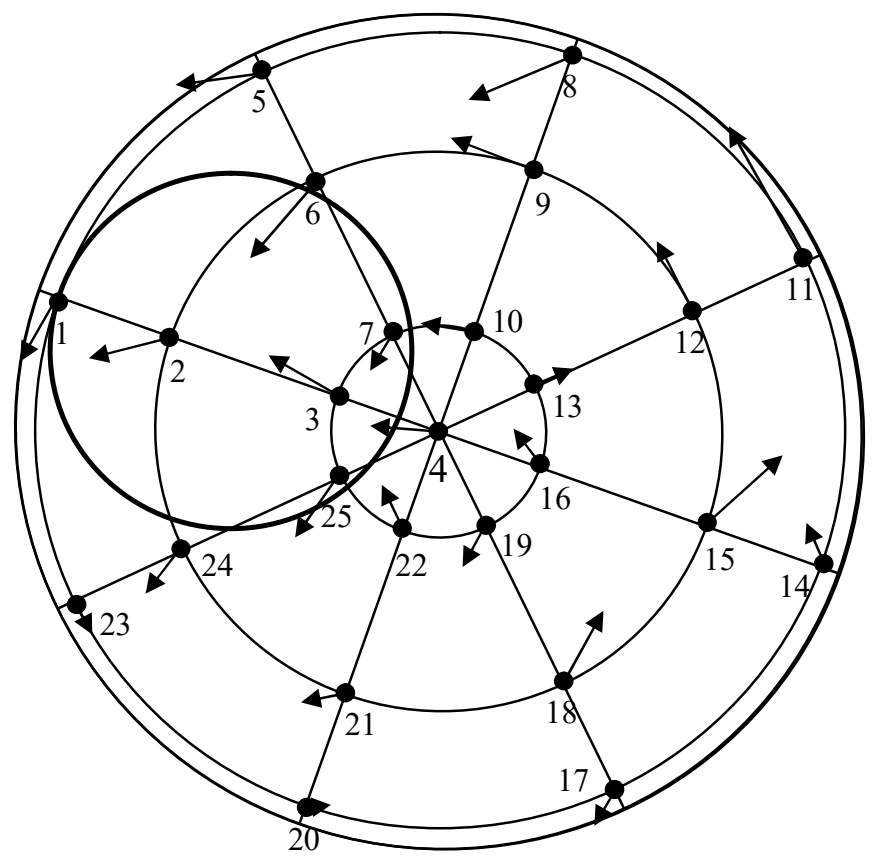

Fig. 4: The Distribution of the Horizontal Component of the Velocity in the Cylinder Head AVL -2 at Z= $80 \mathrm{~mm}, \Delta \mathrm{p}=100 \mathrm{kPa}$. 
The distribution of the horizontal velocity component in the cylinder of the AVL -2 head at $\mathrm{Z}=80 \mathrm{~mm}, \Delta \mathrm{p}=$ $100 \mathrm{kPa}$ is shown in Fig. 4.

Table 2. Components of the velocity, in pixels, measured from the valve slit of the head of AVL -2 , with $\Delta \mathrm{p}=100 \mathrm{kPa}$.

\begin{tabular}{|c|c|c|c|c|}
\hline No. points & $v_{\mathrm{r}}, \mathrm{m} / \mathrm{s}$ & $v_{\varphi .}, \mathrm{m} / \mathrm{s}$ & $v_{z}, \mathrm{~m} / \mathrm{s}$ & v, $\mathrm{m} / \mathrm{s}$ \\
\hline 1 & - & - & - & unavailable \\
\hline 2 & - & - & - & unavailable \\
\hline 3 & 05.38 & -06.11 & 46.33 & 47.04 \\
\hline 4 & 27.01 & -01.52 & 60.69 & 66.45 \\
\hline 5 & 63.71 & 86.48 & 61.00 & 123.53 \\
\hline 6 & 69.52 & 08.36 & 43.89 & 82.64 \\
\hline 7 & 56.57 & -09.75 & 65.65 & 87.21 \\
\hline 8 & 50.25 & -46.50 & 55.44 & 88.10 \\
\hline 9 & 00.01 & -27.82 & 49.81 & 57.05 \\
\hline 10 & -22.24 & 25.54 & 39.80 & 52.26 \\
\hline
\end{tabular}

Figure 5 shows the results of measuring the horizontal velocity component (Table 2) in direction and magnitude in the valve slit in the cylinder of the AVL - 2 head.

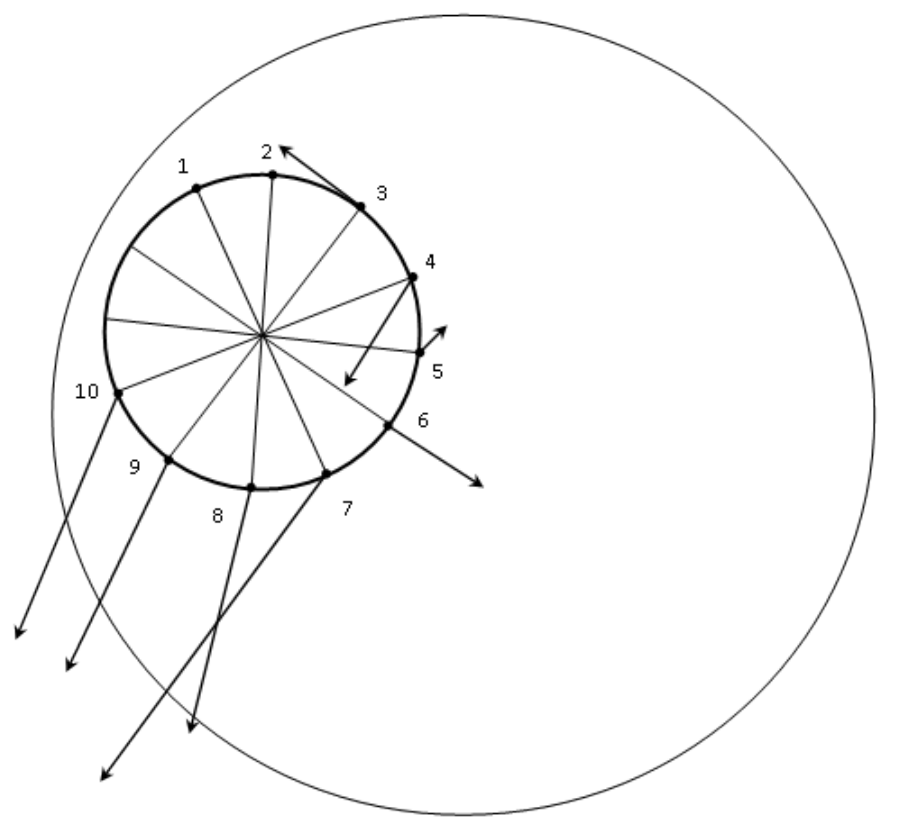

Fig. 5: The Distribution of the Horizontal Components of the Velocities around the Valve Slit When $p=100$ kPa.

\section{Summary}

A series of tests of the research facility allowed us to draw the following conclusions:

1) Even with the most unfavorable LDA signal parameters - a filling frequency of $85 \mathrm{MHz}$ and a signal duration of $1 \mu \mathrm{s}$, which corresponds to movement at the highest possible speed $(150 \mathrm{~m} / \mathrm{s})$ against the direction of movement of the interference fringes, the relative measurement error is less than $3 \%$.

2) At large signal durations $(\sim 0.5 \mathrm{~m} / \mathrm{s})$, when the measurement time was limited only by the capacity of the $T$ code counter, the potential accuracy of the processing system of about $0.1 \%$ was achieved. This mode corresponded to the "freezing" of a particle in the measuring volume.

\section{Conclusions}

In the course of experimental studies of gas-dynamic flows in the cylinder of an internal combustion engine, it was revealed that the LDA-based system allows one to obtain a reliable picture of the distribution of flows in the cylinder. 


\section{Acknowledgments}

The work is performed according to the Russian Government program of Competitive Growth of Kazan Federal University.

\section{References}

[1] Yanta W.J. Measurement of aerosol size distribution switch a Laser Doppler Velocimeter (LDV). - AIAA Paper, N 705, 1973. P. 1-12.

[2] Tariq S. Durrani, Clive A. Greated. Laser systems in flow measurement. Plenum Press, 1977, 289 pp.

[3] Klochkov V.P., Kozlov L.F., Potikevich I.V., Soskin M.S. Laser anemometry, remote spectroscopy and interferometry. Kiev, "Naukova Dumka", 1985, 760s.

[4] Trifonov A.P., Shinakov Yu.S. Joint distinction of signals and estimation of their parameters against interference. M.: Radio and communications, 1986, 264c.

[5] Krishtal V.I., Strashinakij C. S., Milovanov V.N., Ramazanov F.F., Gajsin O.V., Zagirov R.G., Alivanov V.V., Yunusov N.B. An automatic two-component doppler anemometer // Instrumtnts and Experimental Techniques. 1993. Vol. 36. No. 6. P. 202 -203.

[6] Krishtal V.I., Ramazanov F.F. The use of ultra-fast ADC-based PSD for the analysis of the stochastic LDA signal. Materials of the interuniversity scientific - practical conference "Automation and information technology." Naberezhnye Chelny, KamPI, 2002-160c. S.48.49.

[7] Ramazanov F.F. Automated system for measuring the parameters of gas flows in technological installations. Abstract of dissertation for the degree of candidate of technical science. Nab. Chelny. KamPI, 2004.20s.

[8] Slavica Ristić, "Laser Doppler Anemometry and its Application in Wind Tunnel Tests", Scientific Technical Review, Vol.LVII, No.3-4, 2007

[9] RISTIĆ, S., PUHARIĆ, M., SREĆKOVIĆ, M., KUTIN, M.: Laser Doppler Anemometry Application in Hydrodynamic Testing, Atti dela Fondazione Giorgio Ronchi, anno 62, jan, 2007, pp.207-218.

[10] Ramazanov F.F., Ramazanov I.F., Ryadninskaya L.F. The study of flows in power plants using a laser Doppler anemometer. "Energy Saving. Science and education". Collection of reports, ed. Doctor of Technical Sciences THEM. Israfilova.-Naberezhnye Chelny: Publishing and Printing Center NCHI K (P) FU, 2017.-79. P. 82-85.

[11] Ramazanov F.F., Ramazanov I.F. Application of a laser Doppler anemometer for studying gas-dynamic flows with a high degree of turbulence // Scientific and Technical Bulletin of the Volga Region. No. 2, 2019. Kazan: 2019. -96. P. 65-68.

[12] Ramazanov F.F., Ramazanov I.F. Evaluation of the accuracy of measuring the parameters of fluid and gas flows with a laser Doppler anemometer // Scientific and Technical Bulletin of the Volga Region. No. 4, 2019. Kazan: 2019. - 200. P. 93-96. 